\title{
Vision-Based Turning Movement Counting at Intersections by Cooperating Zone and Trajectory Comparison Modules
}

\author{
Mohammad Shokrolah Shirazi $^{1}$ and Brendan Morris ${ }^{1}$
}

\begin{abstract}
This work presents a vision-based vehicle tracking system with an improved method for automatically obtaining turning movement counts at intersections. Improved accuracy is obtained for intersections of varying difficulty through cooperation between complimentary counting modules. When vehicle tracking is robust, a typical zone comparison module quickly accounts for predefined image regions. When vehicle tracking is broken due to occlusion or noise, a trajectory comparison module utilizing the least common subsequence distance is able to count the broken trajectories using the typical scene paths. Experimental evaluations during evening peak hours at two different intersections show an $15 \%$ average improvement at two intersections where the trajectory comparison module is in use $22 \%$ of time. Finally, counts of five working days are compared for another intersection in 15 minute intervals to highlight the system's operational utility for turning behavior analysis at high temporal resolution.
\end{abstract}

\section{INTRODUCTION}

An important research effort in Intelligent Transportation Systems (ITS) is the development of automated systems that monitor flow of traffic and generate traffic information. These traffic monitoring systems regularly use computer vision since video is high in information content which enables more concurrent applications than traditional spot sensor such as loop detectors. For example, with vision techniques it is possible to provide flow, speed, vehicle classification, and detection of abnormalities at the same time [1].

Many vision-based traffic monitoring systems have been proposed [1]-[6]. Pioneering work in 1997 presented a realtime tracking system that utilized vehicle sub-features for robustness against occlusion [2]. Fast background subtraction methods have been used to detect moving regions and track vehicles through bipartite graph association [3] and is now a favorite technique for traffic monitoring. Tracking systems have been extended to also classify the road objects. Simple classification between cars and non-cars was performed using the dimensions of detected vehicles [3]. A dispersion parameter has been used to distinguish between vehicles and pedestrians [4]. More detailed distinctions between 8 different vehicle classes has been performed using region shape descriptors [1]. More recently, vision systems have addressed behavior monitoring and understanding. Intersection accident and collision detection has been performed using detection bounding boxes [5] and hidden Markov model chains [6].

Even with increased processing power and improved vision techniques, there are very few works that explicitly

\footnotetext{
${ }^{1}$ M. S. Shirazi and B. Morris are with the Electrical and Computer Engineering Department, University of Nevada, Las Vegas, NV 89154, USA shirazi@unlv.nevada.edu, brendan.morris@unlv.edu
}

address turning movement (TM) counts at intersections. TM counts are a fundamental measurement for transportation engineers. They are used for a variety of intersection analyses, including traffic operations analyses, intersection design, and transportation planning applications [7]. In addition, TM counts are needed for developing optimized traffic signal timings leading to various benefits such as fuel consumption reduction, air pollution reduction, travel time improvement and anticipated vehicle crash reduction [8].

Traditionally, the TM counts are obtained by field observations where technicians observer an intersection and hand count the number of vehicles. Robust automated counting methods are required because manual counting is labor intensive, limited in number of locations, and budgets do not exist for data collection for large projects [8]-[10].

TM count systems for road design should provide high accuracy as well as long time operation for providing average daily traffic patterns [8]. Most TM counting systems use the same basic zone definition framework [11]-[13] and they count a turn based on the zones traversed. The SCOCA system [11] performs hybrid region and feature-based tracking based of background subtraction while also classifying vehicles. TM counts were collected based on entrance and exit zones of the trajectories. These zone techniques are simple but are ineffective when a vehicle is not tracked well resulting in poor zone localization and low accuracy in TM counting.

In this paper, a new vehicle tracking system for TM counting at intersections is presented which benefits high accuracy as well as long time operational usage. TM count accuracy is improved by specifically handling broken trajectories through the cooperation of the standard zone comparison module and a more detailed trajectory comparison module. When tracking is successful, the zone module is used. However, when tracking fails due to noise or occlusion, the trajectory module uses typical paths to characterize the most likely TM. This cooperative counting system improves performance even without the need for implementing more complicated occlusion handling techniques in the tracker. To demonstrate the long term operational usage of our system, the system collects TM counts 12 hours a day over five days of a week to compare the usage characteristics in 15 minute increments.

The paper is organized as follows: Section II presents the vehicle tracking system and Section III describes the cooperating turning count modules, Section IV shows experimental evaluation, and Section V presents concluding remarks. 


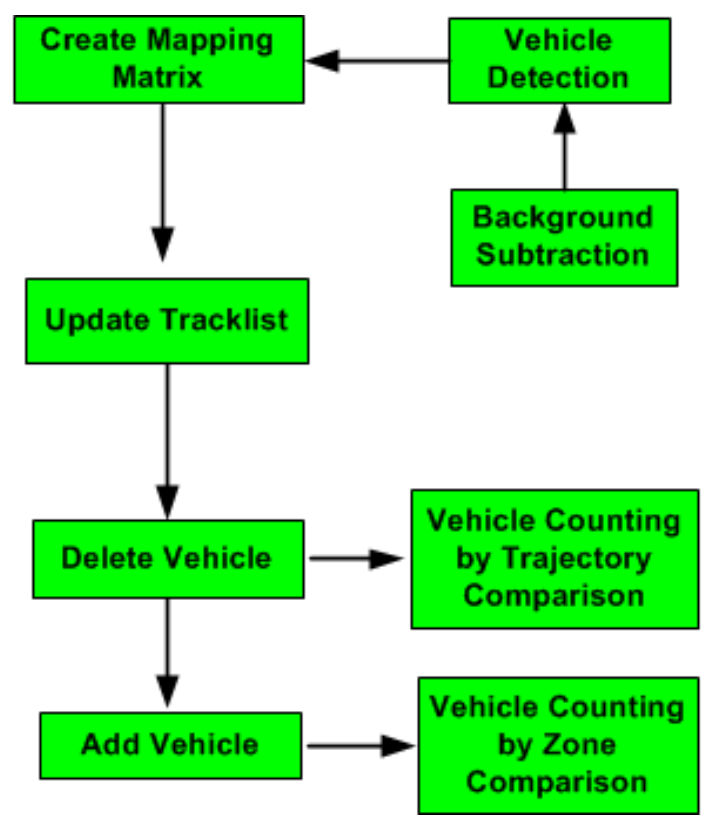

Fig. 1: Vehicle tracking and counting system

\section{VEHICLE TRACKING SYSTEM}

The vehicle tracking and counting system is shown in Fig. 1. Tracking is the key step for intersection analysis and is detailed below.

\section{A. Vehicle Detection}

Vehicle detection is performed using a standard adaptive background subtraction modeling technique. The image background is modeling using Gaussian mixtures [14] to address lighting changes. Moving objects (cars) are detected as pixels that do not fit any of the $K$ background Gaussian models. The foreground image is processed using morphological operations for clean up and each moving regions is characterized through connected component analysis. Each foreground blob has morphological shape measurements taken $m=$ \{centroid, area, perimeter, convex hull area, eccentricity, orientation, best fit ellipse parameters $\}$ for use during tracking.

\section{B. Vehicle Tracking}

Tracking is performed using a detection-track mapping matrix. The mapping matrix is a modification of a bipartite graph to use nearest global matching instead of detection bounding box overlap [15]. This is a two column matrix with detections in the first column and a match from the tracks in the second column.

In order to find a match and update a track, a detection must fit both a dynamic model and an appearance constraint. Vehicle dynamics are modeling using a constant velocity Kalman filter where the state matrix consists of the bounding box and velocity. The predicted location of a track and the centroid of a detection must be within a small error in order for the detection to match the trajectory dynamic model. In addition to matching the dynamics, the appearance of a

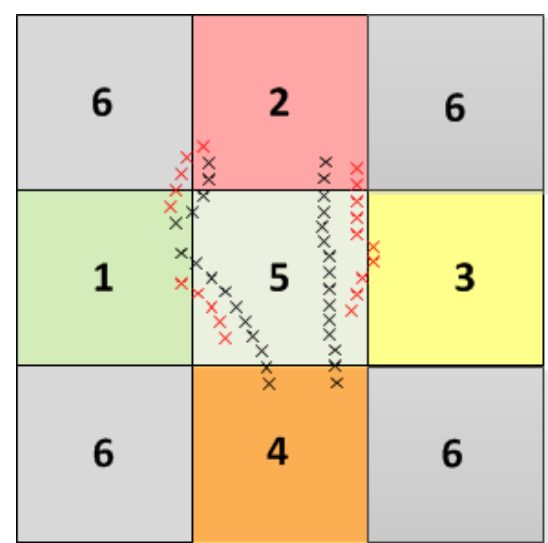

Fig. 2: Complete tracks (black) versus incomplete or undefined tracks (red). The red trajectories cannot be accurately counted using simple zone comparison

detection must match that of a track for association. The appearance model is used to resolve matching ambiguities that may arise during dense traffic situations and occlusions and ensures appearance consistency along a trajectory. The similarity of a trajectory is determined by the $L_{2}$ norm of a detection measurement $m_{d}$ and track $m_{t}$.

\section{Track Maintenance}

Tracks are maintained using a greedy match algorithm that associates the detection that is closest both in dynamics and appearance first. When a detection does not match the existing tracks in the track list, a new track is created. If an existing track does not find a detection for 5 frames, it is marked for deletion. The small frame window allows track numbering to remain consistent even after short disappearances such as during occlusion.

\section{COOPERATING TURNING COUNT MODULES}

TM counting can be improved through cooperation of different counting methods. A zone comparison module examines which image regions are traversed. Unfortunately, this simple method does not work well in the following situations:

1) When a track is incomplete as might occur when two vehicles move from a stop bar in unison and cannot be distinguished until they get separated.

2) When the centroid of trajectory falls into an undefined region due to various noise sources (e.g. occlusion or poor background subtraction).

The trajectory comparison module complements the zone module by addressing these situations by considering the full trajectory for counting purposes.

\section{A. Zone Comparison Module}

Predefined regions in the intersection image, called zones, are defined manually to specify the four cardinal directions \{north, south, east, west\} and the central intersection. The zones are defined based on the set of lines drawn on the stop bars of each intersection direction. A prototype 


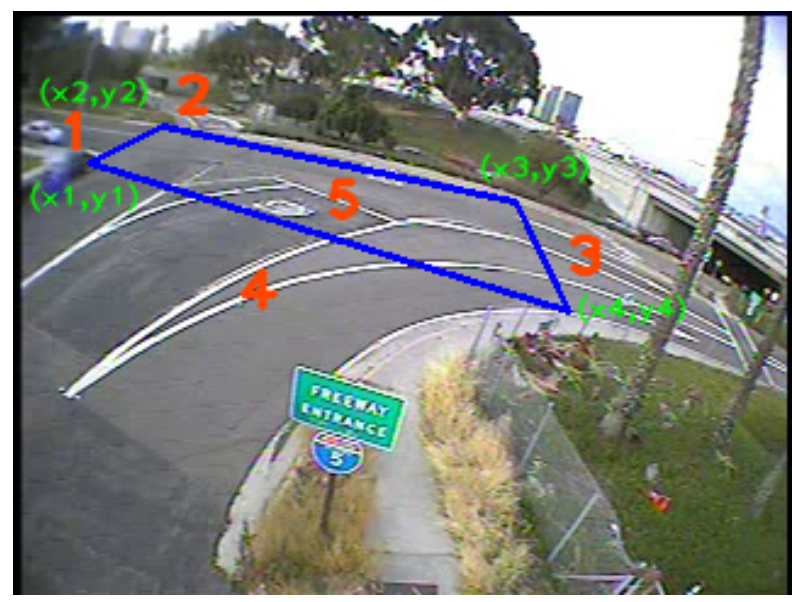

Fig. 3: Intersection 1 zones

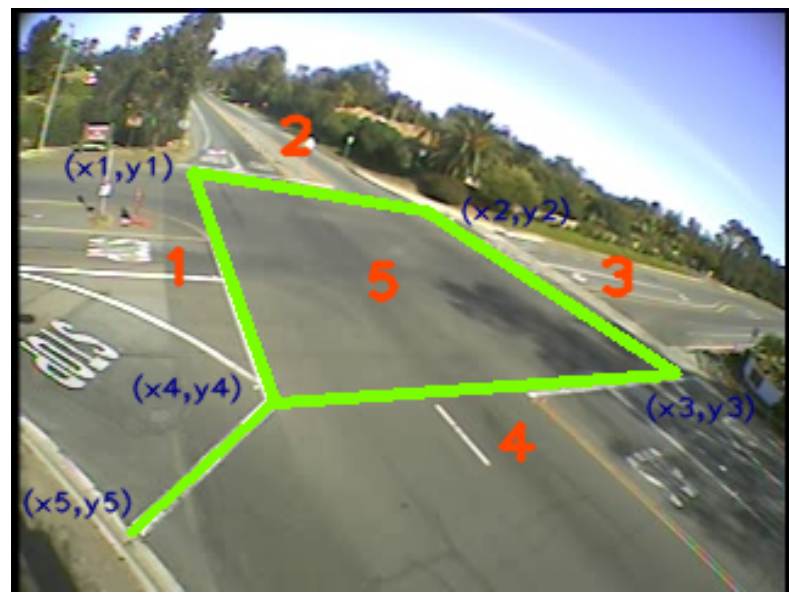

Fig. 4: Intersection 2 zones

intersection is given in Fig. 2. The zones are used to define a regular sequence $(R S)$ set, that is, the set of acceptable zone traversals. For example, $\{1,5,2\}$ indicates a westbound left. During tracking, the vehicle location is mapped to a zone using the set of line equations. The tracker only keeps record or transitions between zones (when the current tracked zone changes) to build the track zone sequence. If the resulting zone sequence exists in the regular sequence set for the intersection, a counter for the associated TM count is incremented. A zone flag is set to indicate if tracking was successful for cooperation with the trajectory module.

Examples of the zones for two intersections are displayed in Figs. 3 and 4. The first intersection (INT1) has 5 regions defined using four coordinates. The second intersection (INT2) also has 5 regions but required an extra line to distinguish the separated right-turn in the lower left of the image. The complete $R S$ set for each of the two intersections is given in Table I. Notice not all TM have a sequence in INT1 and INT2 has also some two zone sequences due to the configuration of the intersection.

\section{B. Trajectory Comparison Module}

Although counting by zone comparison is simple, it is unsuccessful when the obtained sequence of zone numbers is not member of the $R S$. This happens during undesired situations like occlusion. Fig. 2 highlights some of the difficulties when using zone comparison. The trajectories in black are complete and can be counted using zones. The red trajectories all have some issue that would cause the zone module to fail. The top left trajectory has sequence $\{1,6,2\}$ and goes through an undefined region 6 . The bottom left sequence $\{1,5\}$ stops in zone 5 and never goes to an exit zone. Finally, the rightmost track has sequence $\{5,3,5,2\}$, suffering due to occlusion and noisy measurements.

If the $R S$ set cannot be used and the zone flag is not set, the trajectory comparison module will go into effect. This module examines the entire trajectory and compares it with the typical paths in the scene. The typical paths could be learned in a variety of ways such as through clustering observations [1] or even predefined by hand drawing similar to with zone definition. In this work, the paths are defined by the first complete trajectory to traverse each of the entries in the $R S$ set for simplicity.

Trajectories and paths care compared using temporal alignment techniques for similarity measures. These techniques, like longest common substring (LCSS) and dynamic time warping (DTW), have been shown to perform best among popular distance measurement methods for unequal length trajectories [16], [17]. The LCSS distance is utilized due to its robustness to noise and outliers since not all points must be matched. Rather than a one-to-one mapping between all points in trajectories to compare, a point with no good match can be ignored. The LCSS distance [16] can be computed as

$$
D_{L C S S}\left(F_{i}^{T_{i}}, F_{j}^{T_{j}}\right)=1-\frac{\operatorname{LCSS}\left(F_{i}^{T_{i}}, F_{j}^{T_{j}}\right)}{\min \left(T_{i}, T_{j}\right)}
$$

where $T_{i}$ is the length of trajectory $F_{i}$. The LCSS is defined in 1 and represents the number of matching points between two trajectories. $F^{t}=\left\{f_{1}, \ldots, f_{t}\right\}$ denotes the trajectory centroid up to time $t$. The matching requires that compared points are withing a small euclidean distance $\epsilon$ and that the points are not separated by more than $\delta$ samples (to ensure the lengths are comparable). The LCSS distance can be efficiently computed using dynamic programming.

The trajectory comparison module picks up when the zone module fails by comparing the trajectory with all the stored paths of the intersection. The path with the smallest $D_{L C S S}$ value is considered the best match and used to increment the TM counters. Examples of the intersection paths can be seen in Figs. 5 and 6. The paths are color-coded based on their approach zone so all path starting in a particular zone have the same color.

\section{EXPERIMENTAL EVALUATION}

Experimental evaluation was performed in two steps. In the first step, detailed evaluation of the cooperating comparison modules was performed on two different intersections, 
TABLE I: Regular Sequence Set for Turning Movement Directions

\begin{tabular}{|c|c|c|c|c|c|c|c|c|c|c|c|c|}
\hline & WBL & WBT & WBR & NBL & NBT & NBR & EBL & EBT & EBR & SBL & SBT & SBR \\
\hline INT1 & & $\{1,5,3\}$ & $\begin{array}{c}\{1,5,4\} \\
\{2,5,3\}\end{array}$ & $\{2,5,4\}$ & & & & & & & $\begin{array}{c}\{4,5,3\} \\
\{4,3\}\end{array}$ \\
\hline INT 2 & $\{1,5,2\}$ & $\{1,5,3\}$ & $\begin{array}{c}\{1,5,5\} \\
\{1,4\}\end{array}$ & $\{2,5,3\}$ & $\{2,5,4\}$ & $\begin{array}{c}\{2,5,1\} \\
\{2,1\}\end{array}$ & $\{3,5,4\}$ & $\{3,5,1\}$ & $\{3,5,2\}$ & $\{4,5,1\}$ & $\{4,5,2\}$ & $\begin{array}{c}\{4,5,3\} \\
\{3,2\}\end{array}$ \\
\hline
\end{tabular}

$$
\operatorname{LCSS}\left(F_{i}, F_{j}\right)=\left\{\begin{array}{c}
0 \\
1+\operatorname{LCSS}\left(F_{i}^{T_{i}-1}, F_{j}^{T_{j}-1}\right) \\
\max \left(\operatorname{LCSS}\left(F_{i}^{T_{i}-1}, F_{j}^{T_{j}}\right), \operatorname{LCSS}\left(F_{i}^{T}, F_{j}^{T_{j}-1}\right)\right)
\end{array}\right.
$$

$$
\begin{gathered}
T_{i}=0 \mid T_{j}=0 \\
d_{E}\left(f_{T_{i}}, f_{T_{j}}<\epsilon\right) \&\left|T_{i}-T_{j}\right|<\delta \\
\text { otherwise }
\end{gathered}
$$

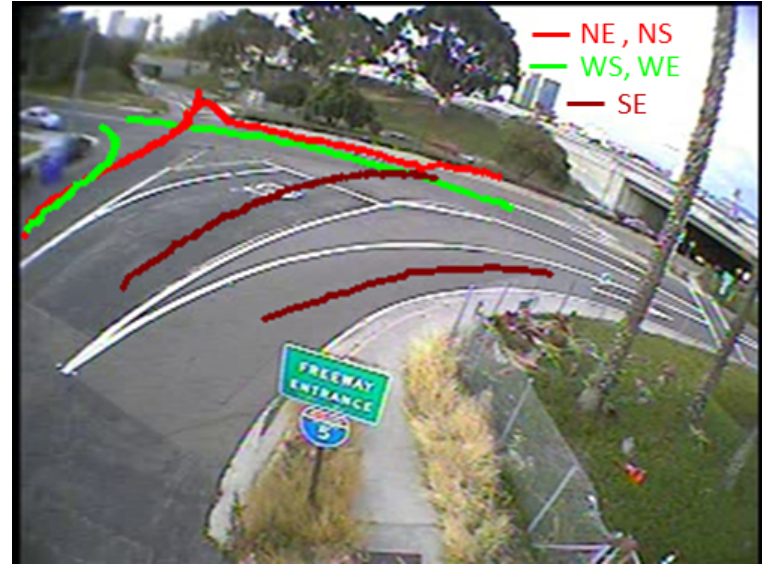

Fig. 5: Typical paths for first intersection (INT 1)

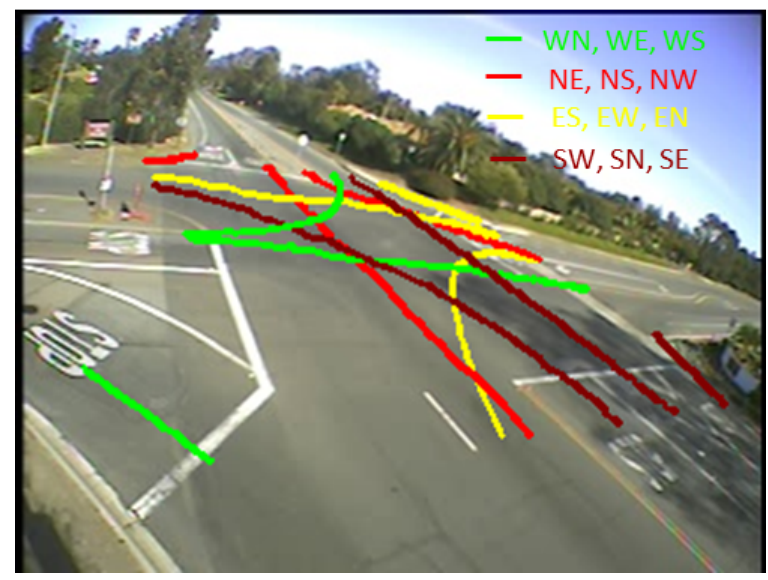

Fig. 6: Typical paths for second intersection (INT 2)

INT1 and INT2. Each intersection was setup by defining zones and paths before examining the TM counts over a two hour period. As is typical in transportation studies, the counts are aggregated into 15 minute intervals to determine the counting accuracy rate. The accuracy rate [9] is defined as

$$
a r=\frac{|M-C|}{M},
$$

where $M$ specifies the manual count and $C$ the automatic count.

\section{A. Learning Paths}

For simplicity, the paths were learned by recording the first full trajectory in each of the TM directions. These full trajectories were selected based on the zone comparison flag and were retained in full frame resolution for later LCSS path comparison. The paths for the experiments are shown in Figs. 5 and 6. The typical path starting from North, West, South, and East zones have red, green, brown, and yellow colors respectively. Notice the high degree of overlap between WE and NE paths in INT1 which makes automatic counting difficult with either zones or trajectories. Also, INT1 is only a three-way intersection and does not have East-bound traffic.

\section{B. Vehicle Tracking \& Counting}

The cooperative TM system was implemented in $\mathrm{C}++$ using OpenCV 2.3 and operates in real-time on an Intel i7. At first evaluation step, our system was evaluated for two different intersections for eight intervals of 13500 frames (15 minutes interval and 2 hour in total). Finally, our system was run for longer time (12 hours of five days in total) with 15 minutes interval to show operational usage as well as high temporal resolution. The GMM background subtraction scheme used $K=5$ mixtures.

TABLE II: Manual Counting \Automatic Counting by Zone for INT 1 (4:00-6:00 p.m)

\begin{tabular}{|c|c|c|c|c|c|}
\hline Typical Path & WBT & WBR & NBL & NBT & SBR \\
\hline 4:00-4:15 p.m & $103 \backslash 74$ & $0 \backslash 0$ & $1 \backslash 0$ & $10 \backslash 1$ & $21 \backslash 20$ \\
\hline 4:15-4:30 p.m & $102 \backslash 89$ & $1 \backslash 0$ & $1 \backslash 0$ & $12 \backslash 1$ & $21 \backslash 19$ \\
\hline 4:30-4:45 p.m & $111 \backslash 79$ & $1 \backslash 1$ & $0 \backslash 0$ & $12 \backslash 0$ & $21 \backslash 21$ \\
\hline 4:45-5:00 p.m & $129 \backslash 110$ & $4 \backslash 3$ & $1 \backslash 1$ & $14 \backslash 2$ & $23 \backslash 19$ \\
\hline 5:00-5:15 p.m & $135 \backslash 84$ & $1 \backslash 1$ & $1 \backslash 6$ & $14 \backslash 2$ & $18 \backslash 18$ \\
\hline 5:15-5:30 p.m & $147 \backslash 74$ & $2 \backslash 3$ & $2 \backslash 6$ & $20 \backslash 7$ & $25 \backslash 23$ \\
\hline 5:30-5:45 p.m & $142 \backslash 88$ & $2 \backslash 1$ & $4 \backslash 4$ & $23 \backslash 4$ & $23 \backslash 20$ \\
\hline 5:45-6:00 p.m & $135 \backslash 107$ & $4 \backslash 3$ & $2 \backslash 0$ & $9 \backslash 0$ & $19 \backslash 20$ \\
\hline Total & $1004 \backslash 706$ & $15 \backslash 12$ & $12 \backslash 17$ & $114 \backslash 17$ & $171 \backslash 160$ \\
\hline Difference & 298 & 3 & 5 & 97 & 11 \\
\hline Accuracy rate & $70 \%$ & $80 \%$ & $58 \%$ & $15 \%$ & $94 \%$ \\
\hline
\end{tabular}

Table II gives the accuracy of the zone comparison module on INT1. This is the baseline for comparison. The westbound through (WBT) (green) direction has the most traffic. The errors in this direction are associated with occlusion happening with northbound vehicles. The northbound directions (red) perform the worst by far because they appear furthest away from the camera which results in poor tracking due to 
TABLE III: Manual counting $\backslash$ Automatic counting by Zone+ LCSS for INT 1 (4:00-6:00 p.m)

\begin{tabular}{|c|c|c|c|c|c|}
\hline Typical Path & WBT & WBR & NBL & NBT & SBR \\
\hline 4:00-4:15 p.m & $103 \backslash 95$ & $0 \backslash 0$ & $1 \backslash 0$ & $10 \backslash 9$ & $21 \backslash 22$ \\
\hline 4:15-4:30 p.m & $102 \backslash 103$ & $1 \backslash 0$ & $1 \backslash 0$ & $12 \backslash 12$ & $21 \backslash 20$ \\
\hline 4:30-4:45 p.m & $111 \backslash 101$ & $1 \backslash 0$ & $0 \backslash 0$ & $12 \backslash 10$ & $21 \backslash 23$ \\
\hline 4:45-5:00 p.m & $129 \backslash 120$ & $4 \backslash 5$ & $1 \backslash 1$ & $14 \backslash 13$ & $23 \backslash 21$ \\
\hline 5:00-5:15 p.m & $135 \backslash 115$ & $1 \backslash 1$ & $1 \backslash 6$ & $14 \backslash 14$ & $18 \backslash 19$ \\
\hline 5:15-5:30 p.m & $147 \backslash 116$ & $2 \backslash 3$ & $2 \backslash 6$ & $20 \backslash 14$ & $25 \backslash 28$ \\
\hline 5:30-5:45 p.m & $142 \backslash 119$ & $2 \backslash 1$ & $4 \backslash 4$ & $23 \backslash 18$ & $23 \backslash 23$ \\
\hline 5:45-6:00 p.m & $135 \backslash 126$ & $4 \backslash 3$ & $2 \backslash 0$ & $9 \backslash 8$ & $19 \backslash 21$ \\
\hline Total & $1004 \backslash 895$ & $15 \backslash 14$ & $12 \backslash 17$ & $114 \backslash 98$ & $171 \backslash 177$ \\
\hline Difference & 109 & 1 & 5 & 16 & 6 \\
\hline Accuracy rate & $89 \%$ & $93 \%$ & $58 \%$ & $86 \%$ & $96 \%$ \\
\hline
\end{tabular}

instability of the blob appearance at north area. The results using both zones and trajectories is presented in Table III. $24 \%$ of the counting is done by the trajectory comparison module for INT1. Overall, the accuracy increased using the LCSS trajectory measure from $63 \%$ to $84 \%$. There were significant improvements in the NBT and WBT directions which indicate how trajectory information can resolve the tracking issues.

The performance from INT2 is given in Table IV. Although the intersection is actually busier than INT1, the camera is in a better position leading to higher quality results. The camera is placed closer to the intersection resulting in higher resolution vehicles and less distortion and overlap between paths. Like in INT1 there is a significant improvement accuracy when using both the zones and LCSS trajectory comparison. In this case, $21 \%$ of counting was by trajectory comparison. In particular, SBL, SBT, and NBT all had accuracy improvements over $20 \%$. Since these were the longest paths with the most traffic, they were prone to occlusion which the trajectory comparison module was able to handle. It is noted however, that the accuracy in some of the other directions were decreased by using LCSS. The reason is that a noisy broken trajectory might find wrong match by LCSS. In the WBR, NBR, and SBR directions there were false counts using the trajectories that marginally affected these directions. Overall, the average accuracy rate increased from $83 \%$ to $92 \%$ with the module cooperation which highlights the effectiveness of the proposed system.

For the second evaluation step, the cooperative TM system was compared over a longer time frame to demonstrate the operational utility of this system. This type of continuous operation provides high temporal resolution and detail of behavioral effects. A third intersection was examined over five working days from 8:00 to 20:00 in 15 minute intervals. Figure 7 shows the third intersection and its typical paths while Figs. 8-10 show the TM counts over the 48 intervals in the 12 hour daily observation period.

As it is shown in figure 8 and it was also calculated, the average number of turning lefts for all five days are in the same typical range of 26 to 30 . However, Wednesday has more fluctuation with higher standard deviation. The burst-line nature of left turning should be considered for traffic signal timing. Wednesday also shows couple of peaks

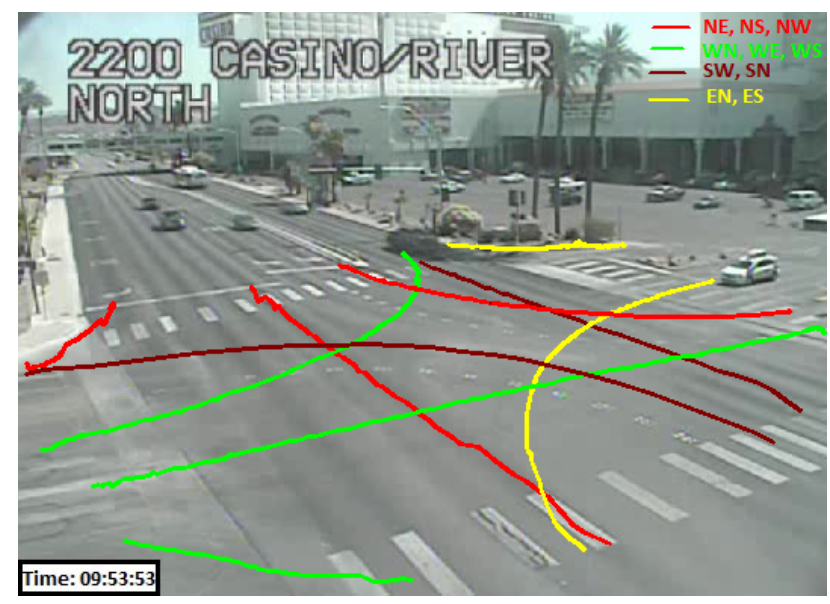

Fig. 7: Typical paths for third intersection (INT 3)

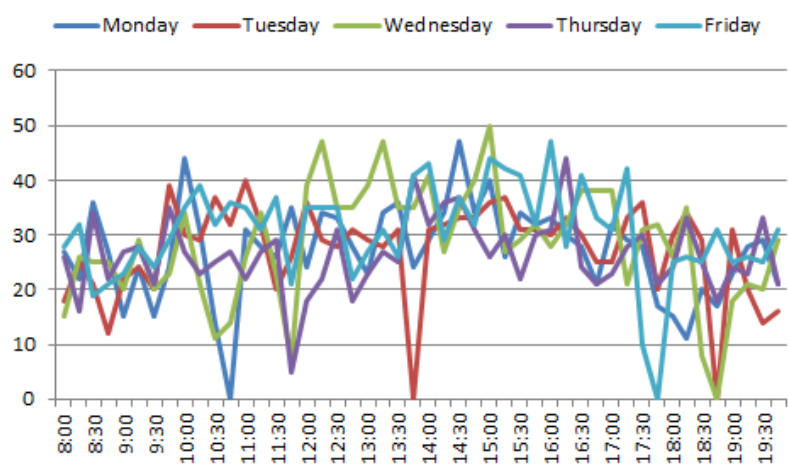

Fig. 8: Turning left counts for INT3

at certain times (15-15:15, 12:15-12:30 and 13:15-13:30) indicating busiest time of the day.

In figure 9, vehicle counts for going straight is shown for each day. Tuesday through Thursday traffic follows a fairly consistent pattern of utilization. Friday, in contrast, has a higher counts all day. The right turn counts, shown in Fig. 10, actually show higher utilization on Tuesday rather than Friday as in the straight through. These subtle count differences cannot be obtained through traditional TM data collection since counts are only sporadically obtained and only during peak travel hours. By continuously monitoring TM counts, it is possible to optimize signal timing.

The three Figs. show some noise measurements (Monday 10:45-11, Tuesday 13:45-14:00, 18:45-19:00, Wednesday 18:45-19:00, Friday 17:45-18:00) where there is a dramatic drop to zero counts. This is a result of the data collection process when the network stream drops a frame and corrupts the time interval. The current implementation stops counting in any interval with a dropped frame.

\section{CONCLUDING REMARKS}

This work presented a vehicle tracking system that is able to obtain accurate turning movement counts at general intersections that has been tested over long time intervals. The system is able to count vehicles for different paths by 
TABLE IV: Manual counting \Automatic counting by Zone and Zone + LCSS for INT 2 (4:00-6:00 p.m)

\begin{tabular}{|c|c|c|c|c|c|c|c|c|c|c|c|c|}
\hline Typical Path & WBLWBT & WBR & NBL & NBT & NBR & EBL & EBT & EBR & SBL & SBT & SBR \\
\hline Manual Counting & 20 & 68 & 164 & 53 & 404 & 36 & 92 & 101 & 73 & 129 & 357 & 62 \\
\hline Zone & 24 & 78 & 160 & 54 & 518 & 38 & 108 & 145 & 77 & 218 & 545 & 71 \\
\hline Zone + LCSS & 24 & 73 & 172 & 58 & 513 & 43 & 99 & 126 & 77 & 218 & 503 & 82 \\
\hline Accuracy rate (Zone) & $80 \%$ & $87 \%$ & $97 \%$ & $98 \%$ & $78 \%$ & $95 \%$ & $85 \%$ & $70 \%$ & $95 \%$ & $59 \%$ & $65 \%$ & $87 \%$ \\
\hline Accuracy rate (Zone+LCSS) & $80 \%$ & $94 \%$ & $92 \%$ & $93 \%$ & $99 \%$ & $87 \%$ & $92 \%$ & $87 \%$ & $100 \%$ & $100 \%$ & $92 \%$ & $85 \%$ \\
\hline
\end{tabular}

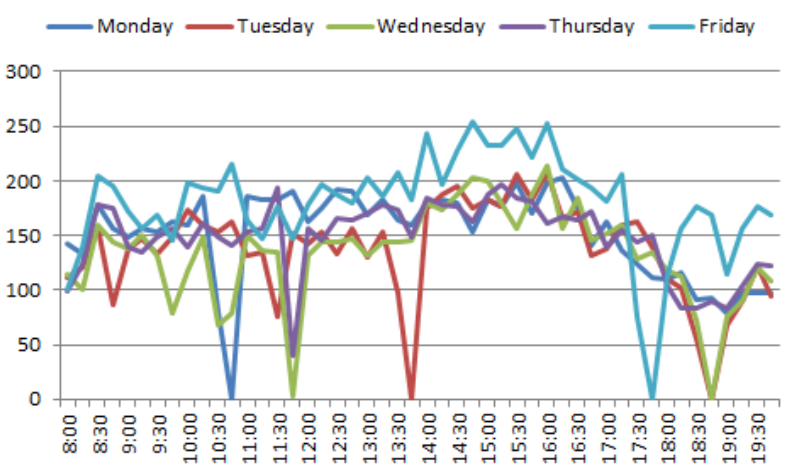

Fig. 9: Going straight counts for INT3

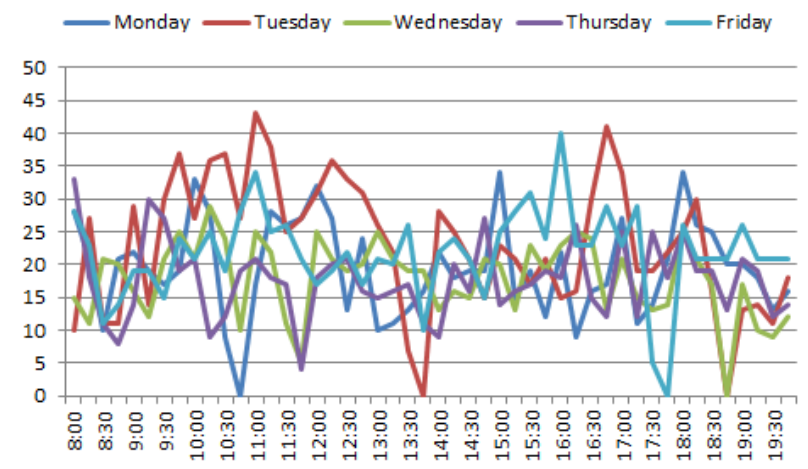

Fig. 10: Turning right counts for INT3

cooperation between two modules called zone comparison and trajectory comparison. The trajectory comparison module uses the LCSS trajectory distance method to complement difficult situations that arise by defining critical zones only. The proposed system improves the counting accuracy by $15 \%$ without implementing specific occlusion handling.

\section{REFERENCES}

[1] B. T. Morris and M. M. Trivedi, "Learning, modeling, and classification of vehicle track patterns from live video," IEEE Trans. Intell. Transp. Syst., vol. 9, no. 3, pp. 425-437, Sep. 2008.

[2] D. Beymer, P. McLauchlan, B. Coifman, and J. Malik, "A real-time computer vision system for measuring traffic parameters," in Proc. IEEE Conf. Computer Vision and Pattern Recognition, San Juan, Puerto Rico, Jun. 1997, pp. 495-501.

[3] S. Gupte, O. Masoud, R. F. K. Martin, and N. P. Papanikolopoulos, "Detection and classification of vehicles," IEEE Trans. Intell. Transp. Syst., vol. 3, no. 1, pp. 37-47, Mar. 2002.

[4] A. J. Lipton, H. Fujiyoshi, and R. S. Patil, "Moving target classification and tracking from real-time video," in Proc. Fourth IEEE Workshop on Applications of Computer Vision, Princeton, New Jersey, Oct. 1998, pp. 8-14.
[5] S. Kamijo, Y. Matsushita, K. Ikeuchi, and M. Sakauchi, "Traffic monitoring and accident detection at intersections," IEEE Trans. Intell. Transp. Syst., vol. 1, no. 2, pp. 108-118, Jun. 2000.

[6] H. Veeraraghavan, O. Maoud, and N. Papanikolopulos, "Computer vision algorithms for intersection monitoring," IEEE Trans. Intell. Transp. Syst., vol. 4, no. 2, pp. 78-89, Jun. 2003.

[7] S. L. Jones, E. D. Arnold, and M.Zanin, "Estimating intersection turning movement," Virginia Transportation Research Council, Tech. Rep. VTRC 99-TAR8, May 1999.

[8] N. R. Bostrom and S. Thompson-Graves, "Turning movement estimation guidelines," Kentucky Transportation Cabinet Departments of Highways Division of Transportation Planning, Tech. Rep., Dec. 1997.

[9] R. Scheneider, "Comparison of turning movement count data collection methods for a signal optimization study," URS Corporation, Tech. Rep., May 2011.

[10] S. Swann, "Alburycity comparison of traffic data collection methods," AlburyCity, Tech. Rep., Oct. 2010.

[11] S. Messelodi, C. M. Modena, and M. Zanin, "A computer vision system for the detection and classification of vehicles at urban road intersections," Pattern Analysis and Applications, vol. 8, no. 1-2, pp. 17-31, Sep. 2005.

[12] A. P. Tarko and R. S. Lyles, "Development of a portable video detection system for counting turning vehicles at intersections," Joint Transportation Research Program, Indiana Department of Transportation and Purdue University, Tech. Rep. FHWA/IN/JTRP-2001/18, 2002.

[13] J. Gerken and B. Guy, "Accuracy comparison of non-intrusive, automated traffic volume counting equipment," Albeck Gerken, Inc., Tech. Rep., Oct. 2009.

[14] C. Stauffer and W. E. L. Grimson, "Adaptive background mixture models for real-time tracking," in Proc. IEEE Conf. Computer Vision and Pattern Recognition, Jun. 1999, pp. 246-252.

[15] W. Fang, Y. Zhao, Y. Yuan, and K. Liu, "Real-time multiple vehicles tracking with occlusion handling," in Proc. International Conference on Image and Graphics, San Juan, Puerto Rico, Aug. 2011, pp. 667672.

[16] B. Morris and M. Trivedi, "Learning trajectory patterns by clustering: Experimental studies and comparative evaluation," in Proc. IEEE Conf. Computer Vision and Pattern Recognition, Miami, Florida, jun 2009, pp. $312-319$.

[17] Z. Zhang, K. Huang, and T. Tan, "Comparison of similarity measures for trajectory clustering in outdoor surveillance scenes," in Proc. IEEE Inter. Conf. on Pattern Recognition, 2006, pp. 1135-1138. 